\title{
Intelligence Control System for Landfills Based on Wireless Sensor Network
}

\author{
Qian Zhang ${ }^{1}$, Chuan Huang ${ }^{1, *}$, and Jian Gong ${ }^{1}$ \\ ${ }^{1}$ State Key Laboratory of Coal Mine Disaster Dynamics and Control, Chongqing University, Chongqing 400044, China
}

\begin{abstract}
This paper put forward an intelligence system for controlling the landfill gas in landfills to make the landfill gas (LFG) exhaust controllably and actively. The system, which is assigned by the wireless sensor network, were developed and supervised by remote applications in workshop instead of manual work. An automatic valve control depending on the sensor units embedded is installed in tube, the air pressure and concentration of LFG are detected to decide the level of the valve switch. The paper also proposed a modified algorithm to solve transmission problem, so that the system can keep a high efficiency and long service life.
\end{abstract}

\section{Introduction}

Landfills is a mature disposal of municipal solid waste (MSW) nowadays because of its security and simplicity [1]. Due to the landfill gas (LFG) is the main product from relevant reactions, which consists of four primary components such as methane, carbon dioxide, oxygen and nitrogen. The landfills had a lasting effect that must be supervised over decades [2]. LFG is a promising renewable energy source provided additional incentive particular the methane in it, while the potential hazards associated is inevitable for its flammability [3-4]. Therefore, gas exhausting pipes arranged in landfills for collecting in general [5]. But the gas was pumped always with a uniform pressure, it leads difficultly to ensure the gas sucked evenly or entirely in time. On account of the problems mentioned above, we propose a new scheme aiming to achieve an efficient procedure to control the gas extraction in the landfill. Furthermore, the intelligence approach can be automatic instead of superfluous manual tasks.

Nowadays, with the development of the technology of the sensors and network technology, the devices with the wireless sensors embedded can achieved in engineering [8]. This paper proposed an air valve with a unit of wireless sensors set on the top of tubes, not only for receiving the data in tube, but also for controlling the switch of the suction depending on data analytics. The tools are available for supervision and alarm, which the thresholds can be set by the experts taking into account the landfill typology.

\section{Intelligence Control System}

\subsection{Profile of the Model}

The rationale of the control system proposed in this work is outlined in Figure 1. In general, the key of control part is air valves which will be set on the top of gas tubes with a series of modules. The first stage is data acquisition, data is collected by masses of wireless sensors. The unit consists of sensors can observe the percentage of $\mathrm{CH}_{4}, \mathrm{CO}_{2}, \mathrm{O}_{2}$ and the air pressure in tube in a certain period. The captured data will be transmitted via wireless communication to the terminal. The computer terminal analyzes and generates a control signal on the basis of comparison between the captured data and predefined threshold set by staff. Staff can set and modify the predefined threshold by computer terminal. Then the control signal send back to each unit without delay and microprocessor implemented receive signals for managing the air valve devices. While after the data transfer procedure, data is uploaded and stored in dedicated database server, and is ready for the following processing such as download and analyze by the staff. So the entire process dominates by wireless sensors automatically.

\subsection{Overview of the Algorithm}

\footnotetext{
* Corresponding author: linette0325@163.com
} 


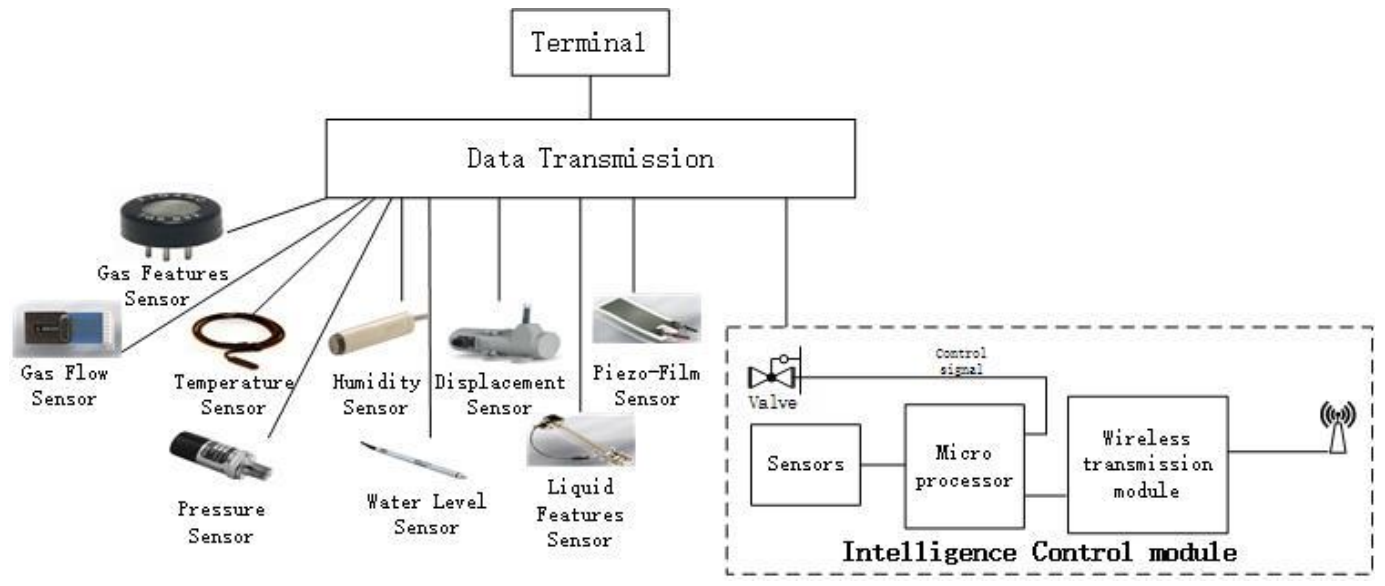

Fig. 1. Components of control network in a landfill.

Despite the wireless sensor units can collect data and receive control signal in a short period of time, the sensors will deal with a large quantity of information in our scenario of landfill in reality. However, each unit consists of several different kinds of sensors, the energy of one unit depending on the battery power and the transmission bandwidth is limited in network. In the previous study by author [11], we found compressive sensing theory demonstrates that it is possible to reduce the number of sampling and reconstruct the original signal accurately with high probability. So the method applied to the scenario to overcome associated problems firstly. However, the herein more detailed key elements of the method must be improved for our system based on wireless sensor networks.

In this paper, the compressive sensing procedure is introduced specific to the problems above. First of all, we use the sparse measurement matrix which the large and dense matrix multiplication operations in data acquisition are replaced by the summation of signal elements in sensor nodes to improve the algorithm. Thus, the storage of the measurement matrix and the computation complexity are decreased. Then, a hard thresholding algorithm with light computationally and high accuracy was proposed to reconstruct the original signal. The process of the proposed approach is provided in detail below.

Each node transmits data gathered to the terminal in a fixed time, while the node saves the original information as a matrix,

$$
\mathrm{X}=\left[\begin{array}{llll}
x_{1} & x_{2} & \mathrm{~L} & x_{\mathrm{N}}
\end{array}\right]^{\mathrm{T}}
$$

where $\mathrm{N}$ is the number of sensors. According to the sparsity $\mathrm{M}$ of the original information, the corresponding number of the measurement obtained and matrixes $\Phi$ generated. The sparse $M \times N$-dimension measurement matrix $\Phi$ with entries is defined as follows

$$
\theta_{i}=\sqrt{\frac{\alpha}{M}} \times\left\{\begin{array}{ccc}
1 & \text { with probability } & \frac{1}{2 \alpha} \\
0 & \text { with probability } & 1-\frac{1}{\alpha} \\
-1 & \text { with probability } & \frac{1}{2 \alpha}
\end{array}\right.
$$

with zero-mean value, $\mathrm{E}\left[\theta_{i}^{2}\right]=\frac{1}{M}$, and $\left|\theta_{i}\right| \leq \sqrt{\frac{\alpha}{M}}$. It is obviously to see that with the value of $\alpha$ increased, the "0" elements increased, and the matrix $\Phi$ will be sparser. This matrix is very easy to compute with only a uniform random generator. For one $M \times N$ matrix, we only have to store a $\frac{M \times N}{2 \alpha}$-dimensional matrix on the sensor.

Measure process in each node is expressed by

$$
\mathrm{Y}=\Phi \mathrm{X}
$$

as a result $\mathrm{Y}=\left[\begin{array}{lll}y_{1} & y_{2} \mathrm{~L} & y_{\mathrm{M}}\end{array}\right]$. At last, nodes translates the measurement information $\mathrm{Y}$.

After the transmitting via wireless local area network, Hard Threshold Iteration method is used in terminal to reconstruct each signal of the original $\mathrm{X}$. The proposed algorithm is summarized as follows:

Initialize: $\mathrm{X}_{1}=0$, residual $\mathbf{r}=\mathbf{y}$, iterations $n=0$;

$\mathrm{X}_{n}$ is the result of original signal which converted by CSSA with parameter $m$ that refers to the maximum number; Supports $\Lambda=\varnothing, J=\varnothing$;

While halting criterion false do $n=n+1$;

$$
\begin{gathered}
\mathrm{x}_{n+1}=\mathrm{x}_{n}+\Phi^{\mathrm{T}} \mathrm{r} \\
J \leftarrow \mathrm{M}\left(\mathrm{x}_{n+1}, 2 m\right) \\
T=\operatorname{supp}\left(\mathrm{x}_{n}\right) \cup J \\
\left.b\right|_{T}=\Phi_{T}^{\dagger} y \\
\mathrm{x}_{n+1} \leftarrow \mathrm{M}(b, m) \\
\mathbf{r}_{n e w}=\mathbf{y}-\boldsymbol{\Phi}_{n+1}
\end{gathered}
$$


return $\mathrm{X}=\mathrm{X}_{n}$.

\section{Simulation of Algorithm}

In this part, simulations by Matlab were employed to examine the effectiveness of the proposed scheme. We analyze the improved transmission algorithm in a laptop with an Intel i5-3210M CPU, 8GB RAM and Windows 10 operating system. First of all, our modified sparse measurement matrix was simulated by comparison with random Gaussian matrix and sparse binary matrix which used in compressed sensing algorithm usually nowadays. We test the simulation with the data length $\mathrm{N}=1024$, measurements $\mathrm{M}=4 \mathrm{~K}$ ( $\mathrm{K}$ represents the sparse of signal), $\mathrm{D}=4$, and $\alpha=\mathrm{M} / 4$ for 1000 times. The results are shown in Table 1, where the space complexity refers to the storage space. The processing time is the interval from the generation.

Table 1. Comparison of complexity of the matrixes

\begin{tabular}{|c|c|c|}
\hline & $\begin{array}{c}\text { Space } \\
\text { complexity } \\
\text { (bytes) }\end{array}$ & $\begin{array}{c}\text { Processing } \\
\text { time } \\
(\mathrm{s})\end{array}$ \\
\hline Random Gaussian matrix & 2990080 & 0.0103 \\
\hline Sparse binary matrix & 2990080 & 0.0058 \\
\hline Modified sparse matrix & 32768 & 0.0007 \\
\hline
\end{tabular}

From Table 1, the storage of random Gaussian matrix and sparse binary matrix can easily overflow on some small memory node. The modified sparse matrix considerably reduces the need of memory size. Furthermore, the averages results of processing time over 1000 times can be seen that the capacity of our proposed matrix outstrips the others significantly.

To quantitatively evaluate the proposed reconstruction method, we simulate experiment for the performance of algorithm the algorithm in terms of root mean square error (RMSE). The RMSE denotes the root mean square error estimated between the original signal and the recovery result. Final result is the mean value of a large number of iterations. The analysis of performance comparison in terms of RMSE is shown. Computational complexity is calculated by these parameters: 1) the length of test data is 1600, 2) according to the randomness in the process, convergence time is an average value of 500 times. Figure 2 reflects the accuracy of the proposed method.

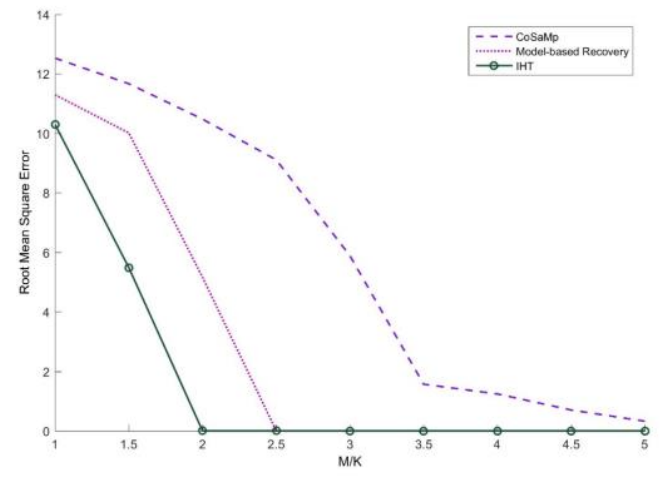

Fig. 2. Comparison of different algorithms. CoSaMp (dashed), Model-based Recovery (dash-dotted) and our method IHT (solid -ring).

Experiments results beyond these algorithms, it can be seen that proposed method can reconstruct the original signal more accurately compared than CoSaMp, Model-based Recovery. Our method can also guarantee reconstruct original signal in a higher accuracy with less measurements.

\section{Conclusion}

This paper presents an innovative intelligence system for controlling the air pump of the landfills, solved many types of engineering problems, such like LFG can be extracted timely, every air exhausting pipe has its adaptive pressure individually, and it is an automatic control system instead of manual work. The procedure includes two particular features. Firstly, control extraction automatically depending on a variety of wireless sensors units set on the valve, the air pressure and concentration of LFG detected which can serve the flow rate of valve. Staffs pay more attention to the result display on the screen and make decision to the valves in the landfills. Secondly, CS-based compression was found to WSN due to its lower complexity and best overall energy efficiency. This paper proposed a CSbased system within a sparse measurement matrix and a iterative hard thresholding recovery algorithm, which is expressed to improve the transmission effectively and precisely of mass data. The results show that the modified method effectively in the scenario. In our future work, we will make further improvement on the system to implement controlling and monitoring in reality.

\section{References}

1. Arigala S G, Tsotsis T T, Yortsos Y C, et al. Gas Generation, Transport, and Extraction in Landfills[J]. Journal of Environmental Engineering, 1995, 121(1):33-44.

2. Young A. Mathematical modeling of landfill gas extraction[J].Journal of Environmental Engineering,1989, 115(6): 1073.

3. Peter Kjeldsen, Morton A. Barlaz, Alix P. Rooker, et al. Present and Long-Term Composition of MSW Landfill Leachate: A Review[J]. Critical Reviews 
in Environmental Science \& Technology, 2002, 32(4):297-336.

4. Townsend $\mathrm{T}$ G, Wise $\mathrm{W}$ R, Jain P. OneDimensional Gas Flow Model for Horizontal Gas Collection Systems at Municipal Solid Waste Landfills [J]. Journal of Environmental Engineering, 2005, 131(12): pages. 1716-1723.

5. Stark T D, Choi H. Methane gas migration through geomembranes[C]// 2005:120-125.

6. Tchobanoglous $\mathrm{G}$, Theisen H, Vigil S. Integrated solid waste management: engineering principles and management issues [J]. Water Science \& Technology Library, 1993, 8(1):63-90.

7. Yu L, Batlle F, Carrera J, et al. Gas flow to a vertical gas extraction well in deformable MSW landfills.[J]. Journal of Hazardous Materials, 2009, 168(2):1404-1416.

8. E. A. Garich, Wireless, Automated Monitoring for Potential Landslide Hazards, Master Thesis, Texas A\&M University, May 2007.

9. D L Donoho. Compressed sensing [J].IEEE Trans. on Information Theory.2006, 52(4): 1289-1306.

10. Joel Tropp, Anna Gilbert, Signal Recovery from Random Measurements via Orthogonal Matching Pursuit, IEEE Transactions on Information Theory, 2008, 53(12): 4655-4666.

11. Liu G, Zhang Q, et al. Model-Based Adaptive Iterative Hard Thresholding Compressive Sensing in Sensor Network for Volcanic Earthquake Detection [J]. International Journal of Distributed Sensor Networks, 2015, 2015(3). 